\title{
BIFURCATION OF LIMIT CYCLES FROM A n-DIMENSIONAL LINEAR CENTER INSIDE A CLASS OF PIECEWISE LINEAR DIFFERENTIAL SYSTEMS
}

\author{
PEDRO TONIOL CARDIN ${ }^{1}$, TIAGO DE CARVALHO ${ }^{1}$ AND \\ JAUME LLIBRE ${ }^{2}$
}

\begin{abstract}
Let $n$ be an even integer. We study the bifurcation of limit cycles from the periodic orbits of the $n$-dimensional linear center given by the differential system

$$
\dot{x}_{1}=-x_{2}, \quad \dot{x}_{2}=x_{1}, \quad \ldots, \quad \dot{x}_{n-1}=-x_{n}, \quad \dot{x}_{n}=x_{n-1},
$$

perturbed inside a class of piecewise linear differential systems. Our main result shows that at most $(4 n-6)^{n / 2-1}$ limit cycles can bifurcate up to first-order expansion of the displacement function with respect to a small parameter. For proving this result we use the averaging theory in a form where the differentiability of the system is not needed.
\end{abstract}

\section{IntRoduCtion AND STATEMENT OF THE MAIN RESUlT}

Piecewise linear differential systems appear in a natural way in control theory, and in the study of electrical circuits. These systems can present complicated dynamical phenomena such as those exhibited by general nonlinear differential systems. One of the main ingredients in the qualitative description of the dynamical behavior of a differential system is the number and the distribution of its limit cycles.

The goal of this paper is to study, in $\mathbb{R}^{n}$ for all $n$ even, the existence of limit cycles of the control systems of the form

$$
\dot{x}=A_{0} x+\varepsilon F(x),
$$

with $|\varepsilon| \neq 0$ a sufficiently small real parameter, where $A_{0}$ is equal to

$$
A_{0}=\left(\begin{array}{ccccccc}
0 & -1 & 0 & 0 & \ldots & 0 & 0 \\
1 & 0 & 0 & 0 & \ldots & 0 & 0 \\
0 & 0 & 0 & -1 & \ldots & 0 & 0 \\
0 & 0 & 1 & 0 & \ldots & 0 & 0 \\
\vdots & \vdots & \vdots & \vdots & \ddots & \vdots & \vdots \\
0 & 0 & 0 & 0 & \ldots & 0 & -1 \\
0 & 0 & 0 & 0 & \ldots & 1 & 0
\end{array}\right)
$$

2010 Mathematics Subject Classification. Primary 34C05, 34A34, 34C14.

Key words and phrases. Limit cycles, bifurcation, control systems, averaging method, piecewise linear differential systems, center. 
and $F: \mathbb{R}^{n} \longrightarrow \mathbb{R}^{n}$ is given by

$$
F(x)=A x+\varphi\left(k^{T} x\right) b,
$$

with $A \in \mathcal{M}_{n}(\mathbb{R}), k, b \in \mathbb{R}^{n} \backslash\{0\}$ and $\varphi: \mathbb{R} \longrightarrow \mathbb{R}$ is the piecewise linear function

$$
\varphi\left(x_{1}\right)=\left\{\begin{aligned}
-1 & \text { if } x_{1} \in(-\infty,-1) \\
x_{1} & \text { if } x_{1} \in[-1,1] \\
1 & \text { if } x_{1} \in(1, \infty)
\end{aligned}\right.
$$

where $x=\left(x_{1}, \ldots, x_{n}\right)^{T}$. The independent variable is denoted by $t$, vectors of $\mathbb{R}^{n}$ are column vectors, and $k^{T}$ denotes a transposed vector.

For $\varepsilon=0$ system (1) becomes

$$
\dot{x}_{1}=-x_{2}, \quad \dot{x}_{2}=x_{1}, \quad \ldots \quad, \dot{x}_{n-1}=-x_{n}, \quad \dot{x}_{n}=x_{n-1} .
$$

We note that the origin of (3) is a global isochronous center, i.e. all its orbits different from the origin are periodic with the same period $2 \pi$. A limit cycle of a differential system is an isolated periodic orbit in the set of all periodic orbits of the system. The Poincaré map (or equivalently, the displacement map) is a suitable tool for studying limit cycles of autonomous systems (detailed explanations can be found in [6] or [8]; see also section 3). We recall that a limit cycle of a system corresponds to an isolated zero of the displacement function.

We must mention that there are many papes studying the bifurcation of limit cycles from the periodic orbits of a center, but almost all of them perturb a 2-dimensional center, see for instance the book [7] and the references quoted there. Of course, there are papers dedicated to perturb centers of dimension $>2$, but not too much. Later on we shall comment some of those papers.

The limit cycles of system (1) for $\varepsilon \neq 0$ sufficiently small that we shall study will be the ones bifurcating from the periodic orbits of th $n$-dimensional center (3) (i.e. of system (1) with $\varepsilon=0$ ). As we shall see later on this study has been made for $n=2,4$. Here we shall do it for $n \geq 6$ even. Our main result can be stated as follows.

Theorem 1. For all $n \geq 6$ even at most $(4 n-6)^{n / 2-1}$ limit cycles of system (1) with $\varepsilon \neq 0$ sufficiently small can bifurcate from the periodic orbits of the n-dimensional center (3), up to first-order expansion of the displacement function of system (1) with respect to the small parameter $\varepsilon$.

Theorem 1 will be proved in section 5 .

In section 2 we shall present the scheme of the proof of Theorem 1, mainly based in four lemmas.

We emphasize that the bifurcation from $\varepsilon=0$ to $\varepsilon \neq 0$ in Theorem 1 takes place for $\varepsilon>0$ and for $\varepsilon<0$ sufficiently small, i.e. on both sides of the value $\varepsilon=0$. We remark that in a Hopf bifurcation the limit cycle only appears on one side of the bifurcation value of the parameter, but in our 
case in which the limit cycles bifurcate from periodic orbits of the period annulus of a center they appear in both sides of $\varepsilon=0$.

The proof of Theorem 1 is based on the first-order averaging method. We will present this method in section 3 , in the form obtained in [1]. The advantage of this result is that the smoothness assumptions for the vector field of the differential system are minimal. In particular, it can be applied to piecewise linear differential systems, which are not $C^{2}$ (not even $C^{1}$ ), as it is required in its classical version, see for instance, Theorem 11.5 of [14]. This non-differential application of the averaging method to control systems was used for the first time in [2]. This method has been used frequently for computing periodic orbits; see for instance [4, 11]. From the paper [3] we can study the stability of the limit cycles of Theorem 1; for more details see remark 16 .

Reference [9] can be seen for a theoretical discussion about suitable transformations of high dimensional differential systems which are small perturbations of a center, into the standard form for averaging. The general idea is to relate this change of variables with the first integrals of the unperturbed center.

We would like to add some comments related to our approach to the problem of counting the limit cycles of piecewise linear differential systems. We have chosen here to study bifurcation with respect to a small parameter from the periodic orbits of a center, up to first-order expansion of the displacement map. For some values of the coefficients, this order is sufficient for finding the exact number of limit cycles. But in some cases the first-order expansion of the displacement map can be identically zero, then a higher order averaging theory is needed. The study can be done by using second-, third-, ... order averaging theory. A key point in these studies is the relation between the averaging theory and the displacement map due to the fact that the displacement map of a piecewise linear differential system is analytic in a neighborhood of a limit cycle.

\section{Scheme of the Proof of Theorem 1}

First in Lemma 2 we shall reduce the $n$ parameters of the vector $b$ in the definition of the function $F(x)$ to one.

Lemma 2. By a linear change of variables system (1) can be transformed into the system

$$
\dot{x}=A_{0} x+\varepsilon \bar{A} x+\varepsilon \varphi\left(x_{1}\right) \bar{b},
$$

where $\bar{A} \in M_{n}(\mathbb{R})$ is an arbitrary matrix and $\bar{b}=\left(b_{1}, \ldots, b_{n}\right)=e_{l}$ for some $l=1,3, \ldots, n-1$, where $e_{l}$ is the $l$-th vector of the canonical basis of $\mathbb{R}^{n}$.

The proof of Theorem 1 uses the averaging theory. One of the main difficulties for applying this theory is to write the differential system that we want to study in the normal form of the averaging theory. For our differential system (1) Lemma 3 provides the change of variables which writes system 
(1) in the normal form of the averaging theory. The change of variables of Lemma 3 is defined using the first integrals of system (1) with $\varepsilon=0$.

Lemma 3. Changing the variables $\left(x_{1}, x_{2}, \ldots, x_{n}\right)$ to $\left(\theta, r, \theta_{1}, r_{1}, \ldots, \theta_{(n / 2-1)}\right.$, $\left.r_{(n / 2-1)}\right)$ by using

$$
\begin{aligned}
x_{1}=r \cos \theta, & x_{2}=r \sin \theta, \\
x_{3}=r_{1} \cos \left(\theta+\theta_{1}\right), & x_{4}=r_{1} \sin \left(\theta+\theta_{1}\right), \\
\vdots & \\
x_{n-1}=r_{(n / 2-1)} \cos \left(\theta+\theta_{(n / 2-1)}\right), & x_{n}=r_{(n / 2-1)} \sin \left(\theta+\theta_{(n / 2-1)}\right),
\end{aligned}
$$

system (4) is transformed into a system of the form

$$
\begin{gathered}
\frac{d r}{d \theta}=\varepsilon H_{1}\left(\theta, r, \theta_{1}, r_{1}, \ldots, \theta_{(n / 2-1)}, r_{(n / 2-1)}\right)+O\left(\varepsilon^{2}\right) \\
\frac{d r_{1}}{d \theta}=\varepsilon H_{2}\left(\theta, r, \theta_{1}, r_{1}, \ldots, \theta_{(n / 2-1)}, r_{(n / 2-1)}\right)+O\left(\varepsilon^{2}\right), \\
\frac{d \theta_{1}}{d \theta}=\varepsilon H_{3}\left(\theta, r, \theta_{1}, r_{1}, \ldots, \theta_{(n / 2-1)}, r_{(n / 2-1)}\right)+O\left(\varepsilon^{2}\right), \\
\vdots \\
\frac{d r_{(n / 2-1)}}{d \theta}=\varepsilon H_{n-2}\left(\theta, r, \theta_{1}, r_{1}, \ldots, \theta_{(n / 2-1)}, r_{(n / 2-1)}\right)+O\left(\varepsilon^{2}\right), \\
\frac{d \theta_{(n / 2-1)}}{d \theta}=\varepsilon H_{n-1}\left(\theta, r, \theta_{1}, r_{1}, \ldots, \theta_{(n / 2-1)}, r_{(n / 2-1)}\right)+O\left(\varepsilon^{2}\right) .
\end{gathered}
$$

where

$$
\begin{gathered}
H_{1}=\cos \theta F_{1}+\sin \theta F_{2}, \\
H_{j}=\cos \left(\theta+\theta_{\frac{j}{2}}\right) F_{j+1}+\sin \left(\theta+\theta_{\frac{j}{2}}\right) F_{j+2}, \\
H_{j+1}=-\frac{1}{r} \cos \theta F_{2}+\frac{1}{r} \sin \theta F_{1}+\frac{1}{r_{\frac{j}{2}}} \cos \left(\theta+\theta_{\frac{j}{2}}\right) F_{j+2}-\frac{1}{r_{\frac{j}{2}}} \sin \left(\theta+\theta_{\frac{j}{2}}\right) F_{j+1},
\end{gathered}
$$

for $j=2,4, \ldots, n-2$, and for $i=1,2, \ldots, n$ we have that $F_{i}=\left(a_{i 1} \cos \theta+\right.$ $\left.a_{i 2} \sin \theta\right) r+\sum_{l=3}^{\frac{n}{2}+1}\left(a_{i(2 l-3)} \cos \left(\theta+\theta_{l-2}\right)+a_{i(2 l-2)} \sin \left(\theta+\theta_{l-2}\right)\right) r_{l-2}+\varphi(r \cos \theta) b_{i}$.

We take $\varepsilon_{0}$ sufficiently small, $m$ arbitrarily large and

$$
D_{m}=\left\{\left(r, \theta_{1}, r_{1}, \ldots, \theta_{(n / 2-1)}, r_{(n / 2-1)}\right) \in\left(\frac{1}{m}, m\right) \times\left[\mathbb{S}^{1} \times\left(\frac{1}{m}, m\right)\right]^{\frac{n}{2}-1}\right\} .
$$

Then the vector field of system (5) is well defined and continuous on $\mathbb{S}^{1} \times$ $D_{m} \times\left(-\varepsilon_{0}, \varepsilon_{0}\right)$. Moreover the system is $2 \pi$-periodic with respect to variable $\theta$ and locally Lipschitz with respect to variables $\left(r, \theta_{1}, r_{1}, \ldots, \theta_{(n / 2-1)}, r_{(n / 2-1)}\right)$.

We will apply the averaging theory stated in Theorem 6 (see Section 3 ) to system (5). Our next step is to find the corresponding averaged function (8). We will denote it by $h: D_{m} \rightarrow \mathbb{R}^{n-1}, h=\left(h_{1}, \ldots, h_{n-1}\right)^{T}$. 
Lemma 4. The components of the averaged function $h$ are given by

$$
h_{1}=c_{1} r+\sum_{l=3}^{\frac{n}{2}+1}\left(c_{1,(2 l-4)} \cos \theta_{l-2}+c_{1,(2 l-3)} \sin \theta_{l-2}\right) r_{l-2}+b_{1} I_{1}(r),
$$

(6)

$$
\begin{aligned}
h_{j}= & \left(c_{j} \cos \theta_{\frac{j}{2}}+c_{(j+1)} \sin \theta_{\frac{j}{2}}\right) r+ \\
& \sum_{l=3}^{\frac{n}{2}+1}\left(c_{j,(2 l-4)} \cos \left(\theta_{\frac{j}{2}}-\theta_{l-2}\right)+c_{j,(2 l-3)} \sin \left(\theta_{\frac{j}{2}}-\theta_{l-2}\right)\right) r_{l-2}+ \\
& b_{j+1} \cos \theta_{\frac{j}{2}} I_{1}(r), \\
h_{j+1}= & c+\sum_{l=3}^{\frac{n}{2}+1}\left(c_{1,(2 l-3)} \cos \theta_{l-2}-c_{1,(2 l-4)} \sin \theta_{l-2}\right) \frac{r_{l-2}}{r}+ \\
& \left(c_{(j+1)} \cos \theta_{\frac{j}{2}}-c_{j} \sin \theta_{\frac{j}{2}}\right) \frac{r}{r_{\frac{j}{2}}}+ \\
& \sum_{l=3}^{\frac{n}{2}+1}\left(c_{j,(2 l-3)} \cos \left(\theta_{\frac{j}{2}}-\theta_{l-2}\right)-c_{j,(2 l-4)} \sin \left(\theta_{\frac{j}{2}}-\theta_{l-2}\right)\right) \frac{r_{l-2}}{r_{\frac{j}{2}}}- \\
& \frac{b_{j+1}}{r_{\frac{j}{2}}} \sin \theta_{\frac{j}{2}} I_{1}(r),
\end{aligned}
$$

for $j=2,4, \ldots, n-2$, where the coefficients $c^{\prime}$ s are given by

$$
\begin{gathered}
c=\left(a_{12}-a_{21}\right) \pi, \quad c_{1}=\left(a_{11}+a_{22}\right) \pi, \\
c_{j}=\left(a_{(j+1) 1}+a_{(j+2) 2}\right) \pi, \quad c_{(j+1)}=\left(a_{(j+2) 1}-a_{(j+1) 2}\right) \pi, \\
c_{1,(2 l-4)}=\left(a_{1(2 l-3)}+a_{2(2 l-2)}\right) \pi, \quad c_{1,(2 l-3)}=\left(a_{1(2 l-2)}-a_{2(2 l-3)}\right) \pi, \\
c_{j,(2 l-4)}=\left(a_{(j+1)(2 l-3)}+a_{(j+2)(2 l-2)}\right) \pi, \\
c_{j,(2 l-3)}=\left(a_{(j+2)(2 l-3)}-a_{(j+1)(2 l-2)}\right) \pi,
\end{gathered}
$$

for $l=3, \ldots, n / 2+1$, and the coefficients $a_{r s}$ are the elements of the matrix $\bar{A}$ of Lemma 2 .

Lemmas 2, 3 and 4 are proved in Section 4.

Let $f: D \longrightarrow \mathbb{R}^{n}$ be a $C^{1}$ function, with $f(a)=0$, where $D$ is an open subset of $\mathbb{R}^{n}$ and $a \in D$. The point $a$ is a simple zero of $f$ if the Jacobian $J_{f}(a)$ of $f$ at $a$ is not zero.

The following result is a key point for proving Theorem 1 .

Lemma 5. From each periodic orbit of the differential system (3), with $n \geq 2$ even, which corresponds to a simple zero in $D_{m}$ of the function 
$h=\left(h_{1}, \ldots, h_{n-1}\right)$ given in (6), a branch of limit cycles bifurcates for the differential system (4).

In [10] the authors studied the bifurcation of limit cycles from a twodimensional center inside $\mathbb{R}^{n}$. In this case they proved that the maximum number of limit cycles that can bifurcate from the periodic orbits of the linear differential center is 1 , up to first-order expansion of the displacement function of the perturbed system with respect to the small parameter $\varepsilon$.

The main result of [2] states that for $n=4$ at most three limit cycles of the piecewise linear differential system (1) bifurcate from the periodic orbits of system (3), up to first-order expansion of the displacement function of (1) with respect to the small parameter $\varepsilon$. Moreover, this upper bound is reached.

In [5] we study the bifurcation of limit cycles from the periodic orbits of a 2-dimensional and 4-dimensional linear center in $\mathbb{R}^{n}$ perturbed inside a class of discontinuous piecewise linear differential systems.

Now for $n \geq 6$ it is very difficult to obtain the exact number of limit cycles of system (1) that bifurcate from the periodic orbits of system (3). Thus the study of the zeros of the averaged function $h=\left(h_{1}, \ldots, h_{n-1}\right)$ for $n \geq 6$ becomes very difficult with respect to the cases $n=2,4$. So for $n \geq 6$ we can only provide the upper bound given in Theorem 1 for the number of limit cycles that can bifurcate from the periodic orbits of system (3).

The proof of Lemma 5 and Theorem 1 will be the subject of sections 4 and 5 respectively.

\section{First-Order Averaging Method}

The aim of this section is to present the first-order averaging method as obtained in [1]. Differentiability of the vector field is not needed. The specific conditions for the existence of a simple isolated zero of the averaged function are given in terms of the Brouwer degree. In fact, the Brouwer degree theory is the key point in the proof of this theorem. We remind here that continuity of some finite dimensional function is a sufficient condition for the existence of its Brouwer degree (see [12] for precise definitions).

Theorem 6. We consider the following differential system

$$
\dot{x}(t)=\varepsilon H(t, x)+\varepsilon^{2} R(t, x, \varepsilon),
$$

where $H: \mathbb{R} \times D \longrightarrow \mathbb{R}^{n}, R: \mathbb{R} \times D \times\left(-\varepsilon_{f}, \varepsilon_{f}\right) \longrightarrow \mathbb{R}^{n}$ are continuous functions, T-periodic in the first variable, and $D$ is an open subset of $\mathbb{R}^{n}$. We define $h: D \longrightarrow \mathbb{R}^{n}$ as

$$
h(z)=\int_{0}^{T} H(s, z) d s
$$

and assume that:

(i) $H$ and $R$ are locally Lipschitz with respect to $x$; 
(ii) for $a \in D$ with $h(a)=0$, there exists a neighborhood $V$ of a such that $h(z) \neq 0$ for all $z \in \bar{V} \backslash\{a\}$ and $d_{B}(h, V, 0) \neq 0$ (here $d_{B}(h, V, 0)$ denote the Brouwer degree of $h$ at 0$)$.

Then, for $|\varepsilon|>0$ sufficiently small, there exists an isolated T-periodic solution $\psi(., \varepsilon)$ of system $(7)$ such that $\psi(0, \varepsilon) \rightarrow a$ as $\varepsilon \rightarrow 0$.

Here we will need some facts from the proof of Theorem 6. Hypothesis (i) assures the existence and uniqueness of the solution of each initial value problem on the interval $[0, T]$. Hence, for each $z \in D$, it is possible to denote by $x(., z, \varepsilon)$ the solution of $(7)$ with the initial value $x(0, z, \varepsilon)=z$. We consider also the displacement function $\zeta: D \times\left(-\varepsilon_{f}, \varepsilon_{f}\right) \longrightarrow \mathbb{R}^{n}$ defined by

$$
\zeta(z, \varepsilon)=\int_{0}^{T}\left[\varepsilon H(t, x(t, z, \varepsilon))+\varepsilon^{2} R(t, x(t, z, \varepsilon), \varepsilon)\right] d t .
$$

From the proof of Theorem 6 we extract the following facts.

Remark 7. For every $z \in D$ the following relation holds

$$
x(T, z, \varepsilon)-x(0, z, \varepsilon)=\zeta(z, \varepsilon) .
$$

The function $\zeta$ can be written in the form

$$
\zeta(z, \varepsilon)=\varepsilon h(z)+\varepsilon^{2} O(1)
$$

where $h$ is given by (8) and the symbol $O(1)$ denotes a bounded function on every compact subset of $D \times\left(-\varepsilon_{f}, \varepsilon_{f}\right)$. Moreover, for $|\varepsilon|$ sufficiently small, $z=\psi(0, \varepsilon)$ is an isolated zero of $\zeta(., \varepsilon)$.

Note that from Remark 7 it follows that a zero $z$ of the displacement function $\zeta(z, \varepsilon)$ at time $T$ provides initial conditions for a periodic orbit of the system of period $T$. We also remark that $h(z)$ is the displacement function up to terms of order $\varepsilon$. Consequently the zeros of $h(z)$, when $h(z)$ is not identically zero, also provides periodic orbits of period $T$.

For a given systems there is the possibility that the function $\zeta$ is not globally differentiable, but the function $h$ is $C^{1}$ as we shall see in Section 5 . In fact, only differentiability in some neighborhood of a fixed isolated zero of $h$ could be enough. When this is the case, one can use the following remark in order to verify the hypothesis (ii) of Theorem 6 .

Remark 8. Let $h: D \longrightarrow \mathbb{R}^{n}$ be a $C^{1}$ function, with $h(a)=0$, where $D$ is an open subset of $\mathbb{R}^{n}$ and $a \in D$. Whenever $a$ is a simple zero of $h$ then there exists a neighborhood $V$ of a such that $h(z) \neq 0$ for all $z \in \bar{V} \backslash\{a\}$ and $d_{B}(h, V, a) \in\{-1,1\}$.

\section{Proof of Lemma 5}

In this section we prove Lemmas 2, 3 and 4 and we give some preliminary results necessary for the proof of Lemma 5 and Theorem 1. 
Proof of Lemma 2. Since the linear change of variables $x=J y$, with $J$ an invertible matrix, transforms system (1) into

$$
\dot{y}=J^{-1} A_{0} J y+\varepsilon J^{-1} A J y+\varepsilon \varphi\left(k^{T} J y\right) J^{-1} b,
$$

we have to find $J$ such that

$$
\begin{gathered}
J^{-1} A_{0} J=A_{1}, \\
k^{T}=e_{1}^{T} J^{-1}, \\
J^{-1} b=\bar{b} .
\end{gathered}
$$

We denote by $z_{i j}$, for $i, j=1, \ldots, n$ the elements of the matrix $J^{-1}$. Using equations (9) and (10), easy computations show that $J^{-1}$ is given by

$$
\left(\begin{array}{ccccccc}
k_{1} & k_{2} & k_{3} & k_{4} & \ldots & k_{n-1} & k_{n} \\
-k_{2} & k_{1} & -k_{4} & k_{3} & \ldots & -k_{n} & k_{n-1} \\
z_{31} & z_{32} & z_{33} & z_{34} & \ldots & z_{3(n-1)} & z_{3 n} \\
-z_{32} & z_{31} & -z_{34} & z_{33} & \ldots & -z_{3 n} & z_{3(n-1)} \\
\vdots & \vdots & \vdots & \vdots & \ddots & \vdots & \vdots \\
z_{(n-1) 1} & z_{(n-1) 2} & z_{(n-1) 3} & z_{(n-1) 4} & \ldots & z_{(n-1)(n-1)} & z_{(n-1) n} \\
-z_{(n-1) 2} & z_{(n-1) 1} & -z_{(n-1) 4} & z_{(n-1) 3} & \ldots & -z_{(n-1) n} & z_{(n-1)(n-1)}
\end{array}\right)
$$

If we take $z_{r r}=1$, for $r=3,5, \ldots, n-1, z_{i j}=0$ (of course, except for the elements mentioned previously), $k_{1}=b_{1} /\left(b_{1}^{2}+b_{2}^{2}\right)$ and $k_{2}=b_{2} /\left(b_{1}^{2}+b_{2}^{2}\right)$ in the expression of $J^{-1}$, then equation (11) is satisfied with $\bar{b}=e_{1}$. In this case we obtain a matrix $J^{-1}$ whose determinant is $k_{1}^{2}+k_{2}^{2}$.

On the other hand, if we take $z_{31}=z_{53}=\cdots=z_{l(l-2)}=1, z_{r r}=1$, for $r=l+2, l+4, \ldots, n-1, z_{1(l-2)}=k_{(l-2)}=1, z_{1(l-1)}=k_{(l-1)}=0$, $k_{l}=-b_{l} /\left(b_{l}^{2}+b_{(l+1)}^{2}\right), k_{(l+1)}=-b_{(l+1)} /\left(b_{l}^{2}+b_{(l+1)}^{2}\right)$ and $z_{i j}=0$ for the remaining elements, in the expression of $J^{-1}$, then equation (11) is satisfied with $\bar{b}=e_{l}$, where $l=3,5, \ldots, n-1$. In this case we obtain for each $l=3,5, \ldots, n-1$ a matrix $J^{-1}$ whose determinant is $-\left(k_{l}^{2}+k_{(l+1)}^{2}\right)$.

We recall that we work in the hypotheses that neither $k$, nor $b$ is the null vector. So at least one of the above statements (i.e. when $\bar{b}=e_{l}$ for some $l=1,3, \ldots, n-1)$ is well defined. Moreover at least one of the above expressions for the determinant of $J^{-1}$ is nonzero. Hence there exists the change of variables $x=J y$. This completes the proof of Lemma 2 .

Now we prove Lemma 3. 
Proof of Lemma 3. System (4) in the variables $\left(\theta, r, \theta_{1}, r_{1}, \ldots, \theta_{(n / 2-1)}\right.$, $\left.r_{(n / 2-1)}\right)$ becomes

$$
\begin{aligned}
& \dot{\theta}=1+\frac{\varepsilon}{r}\left(\cos \theta F_{2}-\sin \theta F_{1}\right), \\
& \dot{r}=\varepsilon H_{1}\left(\theta, r, \theta_{1}, r_{1}, \ldots, \theta_{(n / 2-1)}, r_{(n / 2-1)}\right), \\
& \dot{r_{1}}=\varepsilon H_{2}\left(\theta, r, \theta_{1}, r_{1}, \ldots, \theta_{(n / 2-1)}, r_{(n / 2-1)}\right), \\
& \dot{\theta_{1}}=\varepsilon H_{3}\left(\theta, r, \theta_{1}, r_{1}, \ldots, \theta_{(n / 2-1)}, r_{(n / 2-1)}\right), \\
& \vdots \\
& \dot{r}_{(n / 2-1)}=\varepsilon H_{n-2}\left(\theta, r, \theta_{1}, r_{1}, \ldots, \theta_{(n / 2-1)}, r_{(n / 2-1)}\right), \\
& \dot{\theta}_{(n / 2-1)}=\varepsilon H_{n-1}\left(\theta, r, \theta_{1}, r_{1}, \ldots, \theta_{(n / 2-1)}, r_{(n / 2-1)}\right) .
\end{aligned}
$$

We note that for $|\varepsilon|$ sufficiently small $\dot{\theta}(t)>0$ for each $t$ when $\left(\theta, r, \theta_{1}\right.$, $\left.r_{1}, \ldots, \theta_{(n / 2-1)}, r_{(n / 2-1)}\right) \in \mathbb{S}^{1} \times D_{m}$. Now we eliminate the variable $t$ in the above system by considering $\theta$ as the new independent variable. It is easy to see that the right-hand side of the new system is well defined and continuous on $\mathbb{S}^{1} \times D_{m} \times\left(-\varepsilon_{0}, \varepsilon_{0}\right)$, it is $2 \pi$-periodic with respect to the independent variable $\theta$ and locally Lipschitz with respect to $\left(r, \theta_{1}, r_{1}, \ldots, \theta_{(n / 2-1)}, r_{(n / 2-1)}\right)$. Form (5) is obtained after an expansion with respect to the small parameter $\varepsilon$.

Our next step is to prove Lemma 4. In order to calculate the expression of $h$ given by (6), we will use the following formulas

$$
\begin{gathered}
\int_{0}^{2 \pi} \cos ^{2} \theta d \theta=\pi, \quad \int_{0}^{2 \pi} \sin ^{2} \theta d \theta=\pi, \quad \int_{0}^{2 \pi} \cos (\theta+s) \sin (\theta+s) d \theta=0 \\
\int_{0}^{2 \pi} \cos \theta \cos (\theta+s) d \theta=\pi \cos s, \quad \int_{0}^{2 \pi} \cos \theta \sin (\theta+s) d \theta=\pi \sin s \\
\int_{0}^{2 \pi} \sin \theta \cos (\theta+s) d \theta=-\pi \sin s, \quad \int_{0}^{2 \pi} \sin \theta \sin (\theta+s) d \theta=\pi \cos s \\
\int_{0}^{2 \pi} \cos ^{2}(\theta+s) d \theta=\pi, \quad \int_{0}^{2 \pi} \sin ^{2}(\theta+s) d \theta=\pi, \quad \int_{0}^{2 \pi} \cos \theta \sin \theta d \theta=0 \\
\int_{0}^{2 \pi} \cos \left(\theta+s_{1}\right) \sin \left(\theta+s_{2}\right) d \theta=\pi \sin \left(s_{2}-s_{1}\right) \\
\int_{0}^{2 \pi} \sin \left(\theta+s_{1}\right) \cos \left(\theta+s_{2}\right) d \theta=-\pi \sin \left(s_{2}-s_{1}\right) \\
\int_{0}^{2 \pi} \sin \left(\theta+s_{1}\right) \sin \left(\theta+s_{2}\right) d \theta=\int_{0}^{2 \pi} \cos \left(\theta+s_{1}\right) \cos \left(\theta+s_{2}\right) d \theta= \\
=\pi \cos \left(s_{2}-s_{1}\right)
\end{gathered}
$$

for all $s, s_{1}, s_{2} \in \mathbb{R}$. 
For each $r>0$ we define

$$
\begin{aligned}
& I_{1}(r)=\int_{0}^{2 \pi} \varphi(r \cos \theta) \cos \theta d \theta \\
& I_{2}(r)=\int_{0}^{2 \pi} \varphi(r \cos \theta) \sin \theta d \theta
\end{aligned}
$$

where $\varphi$ is the piecewise linear function given by (2).

The next lemma is proved in [2].

Lemma 9. The integrals $I_{1}$ and $I_{2}$ satisfy

$$
I_{2}(r)=0 \text { for all } r>0,
$$

and

$$
I_{1}(r)= \begin{cases}\pi r & \text { if } 0<r \leq 1, \\ \pi r+\frac{2}{r} \sqrt{r^{2}-1}-2 r \arctan \left(\sqrt{r^{2}-1}\right) & \text { if } r>1 .\end{cases}
$$

Proof of Lemma 4. We must compute

(13) $h_{j}\left(r, \theta_{1}, r_{1}, \ldots, \theta_{(n / 2-1)}, r_{(n / 2-1)}\right)=\int_{0}^{2 \pi} H_{j}\left(\theta, r, \theta_{1}, r_{1}, \ldots, \theta_{(n / 2-1)}, r_{(n / 2-1)}\right) d \theta$,

for $j=1, \ldots, n-1$. Using Lemma 9, from (13) we obtain the expressions given in (6) for the components of the function $h$. This ends the proof of Lemma 4.

For each point $\left(r^{*}, \theta_{1}^{*}, r_{1}^{*}, \ldots, \theta_{(n / 2-1)}^{*}, r_{(n / 2-1)}^{*}\right) \in D_{m}$ of system (6) we have a periodic orbit of system (3), because

$r=\sqrt{x_{1}^{2}+x_{2}^{2}}, \quad r_{i}=\sqrt{x_{(2 i+1)}^{2}+x_{(2 i+2)}^{2}}, \quad \theta_{i}=\arctan \frac{x_{(2 i+2)}}{x_{(2 i+1)}}-\arctan \frac{x_{2}}{x_{1}}$,

for $i=1, \ldots, n / 2-1$ are first integrals of system (3).

Of course any isolated $2 \pi$-periodic solution of (5) with $|\varepsilon| \neq 0$ sufficiently small, corresponds to a limit cycle of (4).

Proof of Lemma 5. Lemma 3 states that the hypotheses of Theorem 6 are fulfilled for system (5), where the function $h$ is given by (6). Using also Remark 8 we conclude that for $|\varepsilon|$ sufficiently small and for each simple zero $\left(r^{*}, \theta_{1}^{*}, r_{1}^{*}, \ldots, \theta_{(n / 2-1)}^{*}, r_{(n / 2-1)}^{*}\right) \in D_{m}$ of $h$, there exists an isolated $2 \pi$-periodic solution $\varphi(., \varepsilon)$ of system (5) such that $\varphi(0, \varepsilon) \rightarrow\left(r^{*}, \theta_{1}^{*}, r_{1}^{*}, \ldots\right.$, $\left.\theta_{(n / 2-1)}^{*}, r_{(n / 2-1)}^{*}\right)$ as $\varepsilon \rightarrow 0$. In short we have proved the Theorem 5 .

For a proof of the next result see Lemma 3.4 of [2].

Lemma 10. The displacement function of system (4) for the transversal section $x_{2}=0$, written in the coordinates of Lemma 3, has the form

$$
\varepsilon h\left(r, \theta_{1}, r_{1}, \ldots, \theta_{(n / 2-1)}, r_{(n / 2-1)}\right)+O\left(\varepsilon^{2}\right) .
$$




\section{Proof of Theorem 1}

The proof of Theorem 1 will be the subject of this section. We note that in order to find the zeros of $h$ in $D_{m}$ it is sufficient to look for them in $(0, \infty) \times\left[\mathbb{S}^{1} \times(0, \infty)\right]^{\frac{n}{2}-1}$. This is due to the fact that $m$ can be chosen arbitrarily large, and $h$, as well as the transformation of Lemma 3 are $2 \pi$ periodic with respect to the variables $\theta_{1}, \ldots, \theta_{(n / 2-1)}$.

Before proving Theorem 1 for arbitrary $n \geq 6$, we shall provide a more detailed proof for the particular case $n=6$.

Proposition 11. Consider system (6) with $n=6$. Let $h:(0, \infty) \times\left[\mathbb{S}^{1} \times\right.$ $(0, \infty)]^{2} \rightarrow \mathbb{R}^{5}$ be the function $h=\left(h_{1}, h_{2}, h_{3}, h_{4}, h_{5}\right)$ whose components are given by (6). Then

(i) $h$ is of class $C^{1}$;

(ii) the maximum number of isolated zeros of $h$ in $(0, \infty) \times\left[\mathbb{S}^{1} \times(0, \infty)\right]^{2}$ is 324 .

5.1. Proof of Proposition 11. Function $h$ is a composition of some elementary functions and the function $I_{1}$. A direct study of $I_{1}$ shows that it is of class $C^{1}$ on $(0, \infty)$. Thus statement (i) holds. We will divide the proof of the statement (ii) into Lemmas 13, 14 and 15.

The following result will be needed later on. For a proof see Lemma 4.2 of $[2]$.

Lemma 12. We consider the equation

$$
I_{1}(r)=\lambda r, \quad r>0
$$

where $I_{1}$ is given by (12) and $\lambda$ is a real parameter. Then we have the following situations.

(a) If $0<\lambda<\pi$, then (14) has a unique solution $r^{*}>1$.

(b) If $\lambda=\pi$, then (14) has the interval $(0,1]$ as the set of solutions.

(c) If $\lambda>\pi$ or $\lambda \leq 0$, then (14) has no solution. 
Our next goal is to find zeros of $h=0$ when $n=6$. The components of $h$ are given by

$$
\begin{aligned}
(15)= & c_{1} r+\left(c_{1,2} \cos \theta_{1}+c_{1,3} \sin \theta_{1}\right) r_{1}+ \\
h_{1} & +\left(c_{1,4} \cos \theta_{2}+c_{1,5} \sin \theta_{2}\right) r_{2}+b_{1} I_{1}(r) \\
h_{2}= & \left(c_{2} \cos \theta_{1}+c_{3} \sin \theta_{1}\right) r+c_{2,2} r_{1}+ \\
& \left(c_{2,4} \cos \left(\theta_{1}-\theta_{2}\right)+c_{2,5} \sin \left(\theta_{1}-\theta_{2}\right)\right) r_{2}+b_{3} \cos \theta_{1} I_{1}(r), \\
h_{3}= & \left(c+c_{2,3}\right)+\left(c_{1,3} \cos \theta_{1}-c_{1,2} \sin \theta_{1}\right) \frac{r_{1}}{r}+ \\
& \left(c_{1,5} \cos \theta_{2}-c_{1,4} \sin \theta_{2}\right) \frac{r_{2}}{r}+\left(c_{3} \cos \theta_{1}-c_{2} \sin \theta_{1}\right) \frac{r}{r_{1}}+ \\
& \left(c_{2,5} \cos \left(\theta_{1}-\theta_{2}\right)-c_{2,4} \sin \left(\theta_{1}-\theta_{2}\right)\right) \frac{r_{2}}{r_{1}}-\frac{b_{3}}{r_{1}} \sin \theta_{1} I_{1}(r), \\
h_{4}= & \left(c_{4} \cos \theta_{2}+c_{5} \sin \theta_{2}\right) r+\left(c_{4,2} \cos \left(\theta_{2}-\theta_{1}\right)+c_{4,3} \sin \left(\theta_{2}-\theta_{1}\right)\right) r_{1}+ \\
& c_{4,4} r_{2}+b_{5} \cos \theta_{2} I_{1}(r), \\
h_{5}= & \left(c+c_{4,5}\right)+\left(c_{1,3} \cos \theta_{1}-c_{1,2} \sin \theta_{1}\right) \frac{r_{1}}{r}+ \\
& \left(c_{1,5} \cos \theta_{2}-c_{1,4} \sin \theta_{2}\right) \frac{r_{2}}{r}+\left(c_{5} \cos \theta_{2}-c_{4} \sin \theta_{2}\right) \frac{r}{r_{2}}+ \\
& \left(c_{4,3} \cos \left(\theta_{2}-\theta_{1}\right)-c_{4,2} \sin \left(\theta_{2}-\theta_{1}\right)\right) \frac{r_{1}}{r_{2}}-\frac{b_{5}}{r_{2}} \sin \theta_{2} I_{1}(r) .
\end{aligned}
$$

By Lemma 2 we get $\left(b_{1}, b_{3}, b_{5}\right)$ equal to $(1,0,0)$ or $(0,1,0)$ or $(0,0,1)$. Each one of the three next lemmas make reference to one of these cases.

Lemma 13. Consider $\left(b_{1}, b_{3}, b_{5}\right)=(1,0,0)$. So system (15) can have at most 324 zeros.

Proof. Using $h_{2}=h_{4}=0$ we obtain

$$
r_{1}=\frac{f_{1}\left(\theta_{1}, \theta_{2}\right)}{g_{1}\left(\theta_{1}, \theta_{2}\right)} r \quad \text { and } \quad r_{2}=\frac{f_{2}\left(\theta_{1}, \theta_{2}\right)}{g_{1}\left(\theta_{1}, \theta_{2}\right)} r
$$

and we get $r \neq 0$. Consider $\widetilde{r}_{1}=g_{1}\left(\theta_{1}, \theta_{2}\right)^{2} r_{1} / r$ and $\widetilde{r}_{2}=g_{1}\left(\theta_{1}, \theta_{2}\right)^{2} r_{2} / r$.

With the notations $\cos \theta_{1}=x, \sin \theta_{1}=z, \cos \theta_{2}=y, \sin \theta_{2}=w$, multiplying $h_{3}=0$ by $\widetilde{r}_{1}$ and $h_{5}=0$ by $\widetilde{r}_{2}$, we obtain a system with four equations and four unknowns $(x, y, z, w)$ given by

$$
\begin{array}{cc}
\widetilde{h}_{3} & =0 \\
\widetilde{h}_{5} & =0 \\
x^{2}+z^{2}-1 & =0 \\
y^{2}+w^{2}-1 & =0 .
\end{array}
$$

Using the software Mathematica we get that the two first equations of (16) are polynomial functions of degree 9. By Bezout's Theorem (see [13]), the 
polynomial system (16) has at most 324 solutions. Using the first equation of (15) and Lemma 12, to each solution $\left(x^{*}, y^{*}, z^{*}, w^{*}\right) \in(-1,1) \times$ $(-1,1) \times(-1,1) \times(-1,1)$ corresponds, at most, one solution $r^{*}$ of $(14)$ and consequently, one solution $r_{1}^{*}$ and one solution $r_{2}^{*}$.

Lemma 14. Consider $\left(b_{1}, b_{3}, b_{5}\right)=(0,1,0)$. So system (15) can have at most 224 zeros.

Proof. Using $h_{1}=h_{4}=0$ we obtain

$$
r_{1}=\frac{f_{3}\left(\theta_{1}, \theta_{2}\right)}{g_{2}\left(\theta_{1}, \theta_{2}\right)} r \quad \text { and } \quad r_{2}=\frac{f_{4}\left(\theta_{1}, \theta_{2}\right)}{g_{2}\left(\theta_{1}, \theta_{2}\right)} r,
$$

and we get $r \neq 0$. Consider $\widetilde{r}_{1}=g_{2}\left(\theta_{1}, \theta_{2}\right)^{2} r_{1} / r$ and $\widetilde{r}_{2}=g_{2}\left(\theta_{1}, \theta_{2}\right)^{2} r_{2} / r$.

With the notations $\cos \theta_{1}=x, \sin \theta_{1}=z, \cos \theta_{2}=y, \sin \theta_{2}=w$, multiplying $h_{5}=0$ by $\widetilde{r}_{2}$ and considering $\widetilde{h}_{2}=g_{2}\left(\theta_{1}, \theta_{2}\right)^{2} z h_{2} / r+\widetilde{r}_{1} x h_{3}$, we obtain a system with four equations and four unknowns $(x, y, z, w)$ given by

$$
\begin{array}{cc}
\widetilde{h}_{2} & =0 \\
\widetilde{h}_{5} & =0 \\
x^{2}+z^{2}-1 & =0 \\
y^{2}+w^{2}-1 & =0 .
\end{array}
$$

Using the software Mathematica we get that the first and the second equations of (17) are polynomial functions of degree 8 and 7 respectively. By Bezout's Theorem, the polynomial system (17) has at most 224 solutions. Using the second equation of (15) and Lemma 12, to each solution $\left(x^{*}, y^{*}, z^{*}\right.$, $\left.w^{*}\right) \in(-1,1) \times(-1,1) \times(-1,1) \times(-1,1)$ corresponds, at most, one solution $r^{*}$ of (14) and consequently, one solution $r_{1}^{*}$ and one solution $r_{2}^{*}$.

Lemma 15. Consider $\left(b_{1}, b_{3}, b_{5}\right)=(0,0,1)$. So system (15) can have at most 224 zeros.

Proof. Using $h_{1}=h_{2}=0$ we obtain

$$
r_{1}=\frac{f_{5}\left(\theta_{1}, \theta_{2}\right)}{g_{3}\left(\theta_{1}, \theta_{2}\right)} r \quad \text { and } \quad r_{2}=\frac{f_{6}\left(\theta_{1}, \theta_{2}\right)}{g_{3}\left(\theta_{1}, \theta_{2}\right)} r
$$

and we get $r \neq 0$. Consider $\widetilde{r}_{1}=g_{3}\left(\theta_{1}, \theta_{2}\right)^{2} r_{1} / r$ and $\widetilde{r}_{2}=g_{3}\left(\theta_{1}, \theta_{2}\right)^{2} r_{2} / r$.

With the notations $\cos \theta_{1}=x, \sin \theta_{1}=z, \cos \theta_{2}=y, \sin \theta_{2}=w$, multiplying $h_{3}=0$ by $\widetilde{r}_{1}$ and considering $\widetilde{h}_{4}=g_{3}\left(\theta_{1}, \theta_{2}\right)^{2} w h_{4} / r+\widetilde{r}_{2} y h_{5}$, we obtain a system with four equations and four unknowns $(x, y, z, w)$ given by

$$
\begin{array}{cc}
\widetilde{h}_{3} & =0 \\
\widetilde{h}_{4} & =0 \\
x^{2}+z^{2}-1 & =0 \\
y^{2}+w^{2}-1 & =0 .
\end{array}
$$

Using the software Mathematica we get that the first and the second equations of (18) are polynomial functions of degree 7 and 8 respectively. By Bezout's Theorem, the polynomial system (18) has at most 224 solutions. 
Using the fourth equation of (15) and Lemma 12, to each solution $\left(x^{*}, y^{*}, z^{*}\right.$, $\left.w^{*}\right) \in(-1,1) \times(-1,1) \times(-1,1) \times(-1,1)$ corresponds, at most, one solution $r^{*}$ of (14) and consequently, one solution $r_{1}^{*}$ and one solution $r_{2}^{*}$.

5.2. Proof of Theorem 1 for the general case. As for the case $n=6$ we note that the function $h$ is a composition of some elementary functions and the $C^{1}$ function $I_{1}$.

Proof of Theorem 1. Consider $n \geq 6$ and $\bar{b}=e_{1}$. The subsystem, obtained from (6), formed by all the equations $h_{j}=0$, where $j$ is even, is a $(n / 2-$ $1) \times(n / 2-1)$ linear system in the variables $r_{i}, i=1, \ldots, n / 2-1$. By Cramer's Rule we get $r_{i}=\Delta r_{i} / \Delta_{n}$, where $\Delta_{n}$ is the determinant of the matriz $\left(P_{j i}\right)$ with $P_{j i}=P_{j i}\left(y_{j / 2}, w_{j / 2}, y_{i}, w_{i}\right)$, a polynomial of degree 2, being the coefficient of $r_{i}$ in $h_{j}$ and $\Delta r_{i}$ is equal to $\Delta_{n}$ replacing the column $i$ by the vector $\left(B_{j} r\right)$, where $-B_{j}$ is the coefficient of $r$ in $h_{j}$. An easy computation shows that $r_{i}=r f_{i} / \Delta_{n}$ where $f_{i}$ is an appropriated function.

It is easy to see that, for each $n, \operatorname{deg} \Delta_{n}=\left(\operatorname{deg} \Delta_{n-2}\right)+2=n-2$, where $\operatorname{deg} p$ denotes the degree of the polynomial $p$ (note that $\Delta_{n}$ has $n / 2-1$ rows).

Multiplying $h_{j+1}$ by $\left(r_{j / 2}\left(\Delta_{n}\right)^{2}\right) / r$, we obtain a polynomial of degree $2 n-$ 3 in the variables $\left(y_{1}, w_{1}, \ldots, y_{n / 2-1}, w_{n / 2-1}\right)$ where $y_{k}=\cos \theta_{k}$ and $w_{k}=$ $\sin \theta_{k}$ for $k=1, \ldots, n / 2-1$. By Bezout's Theorem, the subsystem, obtained of (6), formed by all the equations $h_{j+1}=0$, where $j+1 \geq 3$ is odd, together with the equations $y_{k}^{2}+w_{k}^{2}-1=0$ for $k=1, \ldots, n / 2-1$ has $(4 n-6)^{n / 2-1}$ solutions $\left(y_{1}^{*}, w_{1}^{*}, \ldots, y_{n / 2-1}^{*}, w_{n / 2-1}^{*}\right)$. Using the first equation of $(6)$ and Lemma 12, for each one of the previous solutions, we obtain at most one $r^{*}$, and consequently at most one $r_{i}^{*}, i=1, \ldots, n / 2-1$.

If $\bar{b}=e_{j}$ we replace $h_{j}$ by $h_{1}$ in the evaluation to obtain $r_{i}, i=1, \ldots, n / 2-$ 1. As consequence we apply the Bezout's Theorem for polynomials of smaller degree. So the number of solutions when $\bar{b} \neq e_{1}$ is smaller.

Remark 16. Using the main result of [3] the stability of the limit cycles associated with the solution $\left(r^{*}, \theta_{1}^{*}, r_{1}^{*}, \ldots, \theta_{(n / 2-1)}^{*}, r_{(n / 2-1)}^{*}\right)$ is given by the eigenvalues of the matrix

$\left.\frac{\partial\left(h_{1}, \ldots, h_{n-1}\right)}{\partial\left(r, \theta_{1}, r_{1}, \ldots, \theta_{(n / 2-1)}, r_{(n / 2-1)}\right)}\right|_{\left(r, \theta_{1}, r_{1}, \ldots, \theta_{(n / 2-1)}, r_{(n / 2-1)}\right)=\left(r^{*}, \theta_{1}^{*}, r_{1}^{*}, \ldots, \theta_{(n / 2-1)}^{*}, r_{(n / 2-1)}^{*}\right)}$.

Acknowledgments. The two first authors are partially supported by a FAPESP-BRAZIL grant 2007/07957-8 and grant 2007/08707-5 respectively. The third author is partially supported by the MICIIN/FEDER grant MTM2008-03437, by AGAUR grant 2009SGR-410 and ICREA Academia.

\section{REFERENCES}

[1] A. Buica And J. Llibre, Averaging methods for finding periodic orbits via Brouwer degree, Bull. Sci. Math. 128 (2004), 7-22. 
[2] A. Buica And J. Llibre, Bifurcation of limit cycles from a four-dimensional center in control systems, International Journal of Bifurcation and Chaos, 15 (2005), 26532662 .

[3] A. Buica, J. Llibre and O. Makarenkov, Asymptotic stability of periodic solutions for nonsmooth differential equations with application to the nonsmooth van der Pol oscillator, SIAM J. Math. Anal. 40 (2009), 2478-2495.

[4] C.A. Buzzi, J. Llibre, J.C. Medrado and J. Torregrosa, Bifurcation of limit cycles from a center in $\mathbb{R}^{4}$ in resonance $1: N$, Dynamical Systems 24 (2009), 123-137.

[5] P.T. CARdin, T. DE CARvalho ANd J. Llibre, Limit cycles of discontinuous piecewise linear differential systems, preprint, 2010.

[6] S.N. Chow And J. Hale, Methods of Bifurcation Theory, Springer-Verlag, Berlin 1982.

[7] C. Christopher And C. LI, Limit cycles in differential equations, Birkhauser, Boston, 2007.

[8] J. Guckenheimer And P. Holmes, Nonlinear Oscillations, Dynamical Systems, and Bifurcation of Vector Fields, Springer-Verlag, Berlin 1983.

[9] M. HAN, K. JiAng AND D. Green JR., Bifurcations of periodic orbits, subharmonic solutions and invariant tori of high-dimensional systems, Nonlinear Analysis 36 (1999), 319-329.

[10] J. Llibre AND A. Makhlouf, Bifurcation of limit cycles from a two-dimensional center inside $\mathbb{R}^{n}$, Nonlinear Analysis 72 (2010), 1387-1392.

[11] J. Llibre AND M.A. TeiXeira, On the stable limit cycle of a weight-driven pendulum clock, Eur. J. Phys. 31 (2010), 1249-1254.

[12] N. G. Lloyd, Degree Theory, Cambridge University Press, 1978.

[13] I. R. Shafarevich, Basic Algebraic Geometry, Springer, 1974.

[14] F. Verhulst, Nonlinear Differential Equations and Dynamical Systems, $2^{\text {nd }}$ edition, Universitext, Springer 1996.

${ }^{1}$ IBILCE-UNESP, CEP 15054-000, S. J. Rio Preto, SÃo Paulo, Brazil

2 Departament de Matemàtiques, Universitat Autònoma de Barcelona, 08913 Bellaterra, Barcelona, Catalonia, Spain

E-mail address: pedrocardin@gmail.com

E-mail address: ti-car@hotmail.com

E-mail address: jllibre@mat.uab.cat 